\title{
Phase inversion correlations analysis for oil-water flow in horizontal pipes
}

Laura Prieto $^{\mathrm{a}}$, Andrés Pinilla a, ${ }^{\mathrm{a},}$, Deisy Becerra ${ }^{\mathrm{a}}$, Paula Pico ${ }^{\mathrm{a}}$, Juan P. Valdés ${ }^{\mathrm{a}}$, Eduardo Pereyrab, Nicolás Ratkovich ${ }^{\mathrm{a}}$.

a. Department of Chemical Engineering, Universidad de los Andes, Bogota, Colombia.

b. McDougall School of Petroleum Engineering. The University of Tulsa, Tulsa, OK 74104, United States

\section{Supporting Information}

Table S.1. Mixture viscosity correlations review.

\begin{tabular}{|c|c|c|}
\hline Author & Correlation & \\
\hline Einstein $(1906)^{1}$ & $\frac{\mu_{\mathrm{e}}}{\mu_{\mathrm{c}}}=1+2.5 \phi$ & (Eq. 1) \\
\hline Taylor $(1936)^{2}$ & $\begin{array}{c}\frac{\mu_{\mathrm{e}}}{\mu_{\mathrm{c}}}=1+2.5 \phi \mathrm{A} \\
\mathrm{A}=\left[\frac{\mu_{\mathrm{c}}+2.5 \mu_{\mathrm{d}}}{2.5 \mu_{\mathrm{c}}+2.5 \mu_{\mathrm{d}}}\right]\end{array}$ & $\begin{array}{l}\text { (Eq. 2) } \\
\text { (Eq. 3) }\end{array}$ \\
\hline $\begin{array}{c}\text { Leviton and } \\
\text { Leighton }(1936)^{3}\end{array}$ & $\begin{array}{c}\frac{\mu_{\mathrm{e}}}{\mu_{\mathrm{c}}}=\exp \left[2.5 \mathrm{~A} *\left(\phi+\phi^{\frac{5}{3}}+\phi^{\frac{11}{3}}\right)\right] \\
\text { A is (Eq. 3) }\end{array}$ & (Eq. 4) \\
\hline $\begin{array}{l}\text { Guth and Simha } \\
\qquad(1936)^{4}\end{array}$ & $\frac{\mu_{\mathrm{e}}}{\mu_{\mathrm{c}}}=1+2.5 \phi+14.1 \phi^{2}$ & (Eq. 5) \\
\hline Vand $(1948)^{5}$ & $\frac{\mu_{\mathrm{e}}}{\mu_{\mathrm{c}}}=\exp \left(\frac{2.5 \phi}{1-0.609 \phi}\right)$ & (Eq. 6) \\
\hline Roscoe $(1952)^{6}$ & $\frac{\mu_{\mathrm{e}}}{\mu_{\mathrm{c}}}=(1-\phi)^{-2.5}$ & (Eq. 7) \\
\hline Vermeulen $(1955)^{7}$ & $\frac{\mu_{\mathrm{e}}}{\mu_{\mathrm{c}}}=\frac{1}{1-\phi}\left[1+\frac{1.5 \phi \mu_{\mathrm{d}}}{\mu_{\mathrm{c}}+\mu_{\mathrm{d}}}\right]$ & (Eq. 8) \\
\hline $\begin{array}{l}\text { Maron-Pierce } \\
(1956)^{8}\end{array}$ & $\frac{\mu_{\mathrm{e}}}{\mu_{\mathrm{c}}}=\left(1-\frac{\phi}{\phi_{\max }}\right)^{-2}$ & (Eq. 9) \\
\hline $\begin{array}{c}\text { Dougherty \& } \\
\text { Krieger }(1959)^{9}\end{array}$ & $\frac{\mu_{\mathrm{e}}}{\mu_{\mathrm{c}}}=\left(1-\frac{\phi}{\phi_{\max }}\right)^{-2.5 \phi_{\max }}$ & (Eq. 10) \\
\hline Eilers $(1941)^{10}$ & $\frac{\mu_{\mathrm{e}}}{\mu_{\mathrm{c}}}=\left[1+2.5 \phi\left(1-\alpha_{\mathrm{E}} \phi\right)^{-1}\right]^{2}$ & (Eq. 11) \\
\hline Thomas $(1965)^{11}$ & $\frac{\mu_{\mathrm{e}}}{\mu_{\mathrm{c}}}=\left[1+2.5 \phi+10.05 \phi^{2}+0.00273 \exp (16.6 \phi)\right]$ & (Eq. 12) \\
\hline Chong $(1971)^{12}$ & $\frac{\mu_{\mathrm{e}}}{\mu_{\mathrm{c}}}=\left[1+0.75 \frac{\phi}{\phi_{\max }}\left(1-\frac{\phi}{\phi_{\max }}\right)^{-1}\right]^{2}$ & (Eq. 13) \\
\hline
\end{tabular}




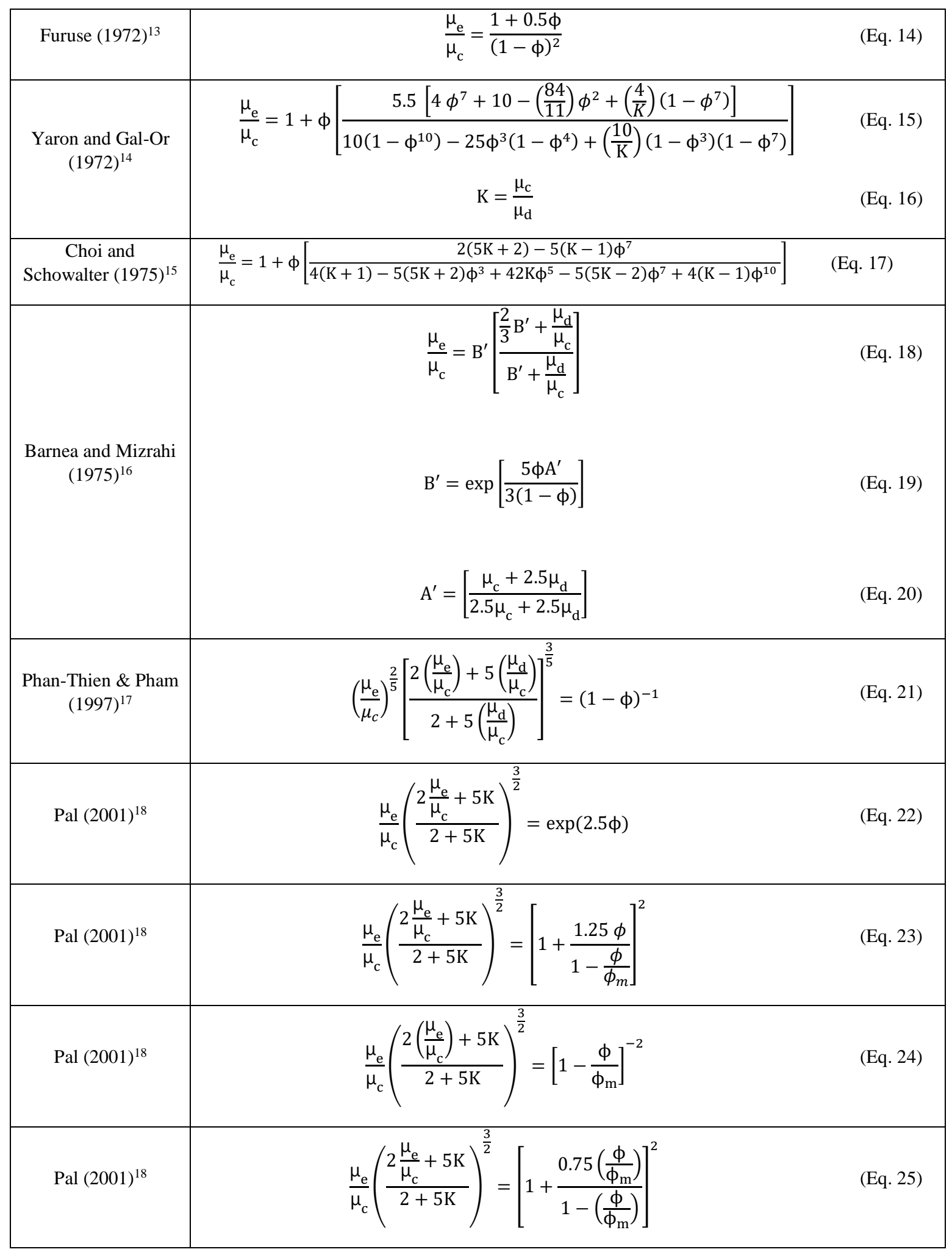

\section{References}

(1) Einstein, A. A New Determination of Molecular Dimensions. Ann. Phys. 1906, 324 (2), 289. 
(2) Taylor, G. I. Correlation Measurements in a Turbulent Flow through a Pipe. Proc. R. Soc. London. Ser. A - Math. Phys. Sci. 1936, 157 (892), 537.

(3) Leviton, A.; Leighton, A. Viscosity Relationships in Emulsions Containing Milk Fat. J. Phys. Chem. 1936, 40 (1), 71.

(4) Guth, E.; Simha, R. Untersuchungen Über Die Viskosität von Suspensionen Und Lösungen. 3. Über Die Viskosität von Kugelsuspensionen. Kolloid-Zeitschrift 1936, 74 (3), 276.

(5) Vand, V. Viscosity of Solutions and Suspensions. I: Theory. J. Phys. Colloid Chem. 1948, 52 (2), 277.

(6) Roscoe, R. The Viscosity of Suspensions of Rigid Spheres. Br. J. Appl. Phys. 1952, 3 (8), 267.

(7) Vermeulen, T.; Williams, G. M.; Langlois, G. E. Interfacial Area in Liquid-Liquid and GasLiquid Agitation. Chem. Eng. Prog. 1955, 51.

(8) Maron, S. H.; Pierce, P. E. Application of Ree-Eyring Generalized Flow Theory to Suspensions of Spherical Particles. J. Colloid Sci. 1956, 11 (1), 80.

(9) Krieger, I. M.; Dougherty, T. J. A Mechanism for Non-Newtonian Flow in Suspensions of Rigid Spheres. Trans. Soc. Rheol. 1959, 3 (1), 137.

(10) Eilers, H. Die Viskisitat von Emulsionene Hochviscoser Stoffe Als Funktion Der Konzentration. Kolloid-Zeitschrift 1941, 97, 271.

(11) Thomas, D. G. Transport Characteristics of Suspension: VIII. A Note on the Viscosity of Newtonian Suspensions of Uniform Spherical Particles. J. Colloid Sci. 1965, 20 (3), 267. https://doi.org/10.1016/0095-8522(65)90016-4.

(12) Chong, J. S.; Christiansen, E. B.; Baer, A. D. Flow of Viscous Fluid through a Circular Aperture. J. Appl. Polym. Sci. 1971, 15 (2), 369.

(13) Furuse, H. Viscosity of Concentrated Solution. Jpn. J. Appl. Phys. 1972, 11 (10), 1537-1541. https://doi.org/10.1143/JJAP.11.1537.

(14) Yaron, I.; Gal-Or, B. On Viscous Flow and Effective Viscosity of Concentrated Suspensions and Emulsions. Rheol. Acta 1972, 11 (3-4), 241.

(15) Choi, S. J.; Schowalter, W. R. Rheological Properties of Nondilute Suspensions of Deformable Particles. Phys. Fluids 1975, 18 (4), 420.

(16) Barnea, E.; Mizrahi, J. A Generalised Approach to the Fluid Dynamics of Particulate Systems Part 2: Sedimentation and Fluidisation of Clouds of Spherical Liquid Drops. Can. J. Chem. Eng. 1975, 53 (5), 461.

(17) Phan-Thien, N.; Pham, D. C. Differential Multiphase Models for Polydispersed Suspensions and Particulate Solids. J. Nonnewton. Fluid Mech. 1997, 72 (2-3), 305.

(18) Pal, R. Single-Parameter and Two-Parameter Rheological Equations of State for Nondilute Emulsions. In Industrial and Engineering Chemistry Research; 2001; Vol. 40, p 5666. 